\title{
ESTUDIO DELPHI SOBRE LA COOPERATIVIZACIÓN DE LOS SERVICIOS SOCIALES EN EL TERRITORIO HISTÓRICO DE GIPUZKOA
}

\author{
POR \\ Enekoitz ETXEZARRETA ETXARRI y \\ Eusebio LASA ALTUNA ${ }^{1}$
}

\section{RESUMEN}

En el presente artículo se muestran los principales resultados obtenidos del estudio Delphi realizado a cargos políticos y técnicos del Departamento de Política Social de la Diputación Foral de Gipuzkoa, sobre la posibilidad de cooperativizar la provisión de los servicios sociales en este territorio histórico. Para ello, el artículo se ha estructurado de dos partes diferenciadas. Una primera desarrolla el marco teórico que sirve de inspiración para el trabajo empírico, donde se apuntan las principales propuestas teóricas que inciden en la dimensión colectiva de la participación ciudadana en la gestión de los servicios públicos. Entre los distintos modelos, se han destacado aquellos que priorizan la participación ciudadana a través de entidades de economía social y solidaria. La segunda parte se dedica al relato de los resultados de la investigación de campo. Para ello, primero se exponen las notas metodológicas que dan cuenta del proceso de preparación del análisis Delphi, para acto seguido sintetizar los principales resultados obtenidos en este estudio. Este trabajo se cierra con un apartado de conclusiones y líneas futuras de trabajo.

Palabras clave: Co-construcción, Co-producción, Welfare Mix, Economía Social y Solidaria, Servicios Sociales.

Claves ECONLIT: D63, H83, I38, P13.

\footnotetext{
${ }^{1}$ Profesores e investigadores de Euskal Herriko Unibertsitatea/Universidad del País Vasco. Instituto de Derecho Cooperativo y Economía Social-GEZKI. Direcciones de correo electrónico: enekoitz.etxezarreta@ehu.es y eusebio.lasa@ehu.es.
}

REVESCO Nº 120 - Primer Cuatrimestre 2016 - ISSN: 1885-8031 - www.ucm.es/info/revesco

http://dx.doi.org/10.5209/rev_REVE.2016.v120.49699

Fecha de recepción: 14/03/2015

Fecha de aceptación: 02/06/2015 


\title{
A DELPHI STUDY ON THE COOPERATIVISATION OF THE SOCIAL SERVICES IN THE HISTORICAL TERRITORY OF GIPUZKOA
}

\begin{abstract}
This article shows the main results obtained from the Delphi study, which was made of politicians and technicians from the Department of Social Policy in the County Council of Gipuzkoa, concerning the possibility of cooperativizing the provision of social services in this historical territory. With this in mind, the structure of this article is in two different parts. The first part develops the theoretical framework which serves as inspiration for the empirical work, where note is made of the main theoretical proposals that have a bearing on the collective dimension of citizen participation in the management of public services. Among the various models, those which prioritise public participation through social and solidarity economy entities stand out. The second part concerns itself with the presentation of the field research results. To this end, the methodological notes concerning the preparation process for the Delphi analysis are presented first and this is immediately followed by a synthesis of the main results obtained in this study. The article ends with a section of conclusions and future lines of action.
\end{abstract}

Keywords: Co-construction, Co-production, Welfare Mix, Social and Solidarity Economy, Social Services.

\section{MARCO TEÓRICO}

\section{1. ¿A quién corresponde la provisión de los servicios sociales?}

¿Cuál debe ser el papel del estado y cual el del mercado en la provisión de los servicios de bienestar? ¿Debe ser el estado, como en el modelo socialdemócrata, el proveedor principal o deben ser estos servicios privatizados y provistos por el mercado, tal y como preconiza la tesis neoliberal? Estas cuestiones son centrales a día de hoy en la mayor parte de los países europeos, y la respuesta generalizada en los últimos tiempos va en la dirección de la progresiva privatización de los servicios.

Sin embargo, ninguna de estas dos posibles estrategias afronta debidamente la cuestión de la participación ciudadana en la provisión de estos servicios. Mejor dicho, cada una de ellas responde de forma diferenciada a este aspecto. La tesis socialdemócrata sitúa el control ciudadano en el uso de los mecanismos de la democracia representativa y la tesis neoliberal

REVESCO No 120 - Primer Cuatrimestre 2016 - ISSN: 1885-8031 - www.ucm.es/info/revesco 
sitúa este control en la deliberación (o capacidad) de compra de los individuos en el mercado. Ninguna de ellas reflexiona sin embargo sobre el papel que debe jugar la sociedad civil como agente activo en la provisión de dichos servicios, y no como mero usuario de los mismos. Tampoco toman en consideración la dimensión colectiva, más allá de la deliberación individual (de voto o de compra), de la propia sociedad civil, que articulada a través de distintas entidades del tercer sector reclama ser tenida en cuenta en estas cuestiones.

Y el hecho es que este tercer sector siempre ha actuado de una forma muy integrada tanto con el estado como con el mercado en la provisión de los servicios públicos (Brandsen y Pestoff, 2008). En algunos países con un rol preponderante, como en Alemania u Holanda, donde han sido pieza fundamental en la construcción de los estados de bienestar de la posguerra. En otros el tercer sector ha despuntado a raíz de la ola liberalizadora de los últimos tres decenios, como puede ser el caso del Reino Unido.

Además de ello, y aunque la literatura sobre la gestión pública lo haya ignorado durante mucho tiempo, iniciativas de participación ciudadana directa en la provisión de servicios básicos han existido desde siempre. Tal y como concluía Ostrom (1999), ningún mercado puede sobrevivir sin una provisión gubernamental de los bienes públicos, pero al mismo tiempo, ningún gobierno puede ser eficiente y equitativo sin un considerable input por parte de la ciudadanía. Este input, esta mayor implicación de los "usuarios", puede canalizarse a través de un sistema democrático de supervisión estatal, o bien a través de entidades del tercer sector (Walzer, 1988).

Esta estrategia de posibilitar servicios sociales más descentralizados y participativos pone en primer plano la dimensión local, en términos territoriales, y el tercer sector o la economía social en términos de gobernanza, al ser ambos espacios (en principio) más proclives a democratizar y a resocializar las estructuras sociales.

En definitiva, esta perspectiva pretende afrontar al mismo tiempo dos retos a través de la misma herramienta: la regeneración democrática de las estructuras públicas por un lado, y la reforma del estado de bienestar por otro, a través de una mayor participación de la economía social. Se considera pues que la participación de la economía social "podría jugar un rol significativo en la renovación de los sistemas políticos democráticos y de la configuración del Estado de Bienestar" (Pestoff; Osborne; Brandsen, 2008: 593) 


\subsection{El "welfare mix" como marco de actuación}

Desde finales de los 80 e inicios de los 90 se generalizaron una serie de políticas públicas en la totalidad de los países europeos que son los que a día de hoy condicionan en gran medida el marco de actuación de las decisiones públicas. Estas políticas buscaban resolver un problema que se planteaba de esta forma: ¿cómo afrontar desde las instituciones públicas una realidad de nuevos riesgos sociales emergentes teniendo en cuenta la restricción impuesta por un sector público hipertrofiado, ineficiente y sin posibilidad de expansión organizativa?

La naturaleza de estos nuevos riesgos cuestionaba profundamente, a su vez, la eficiencia de los tradicionales mecanismos de trasferencia de ingresos ofertados por los estados de bienestar (tales como la seguridad social, el sistema de pensiones, etc.) y reorientaba las políticas hacia la prioridad de desplegar una amplia red de servicios sociales (Ascoli y Ranzi, 2002).

La solución a esta disyuntiva fue adoptar políticas privatizadoras que como afirman Ascoli y Ranzi (2002) incidían en dos direcciones: i) la reorganización del sistema público a través de la introducción de mecanismos de mercado en la gestión de lo público (New Public Management), y; ii) la intensa y directa incorporación de agentes privados y no-lucrativos en la oferta de servicios de bienestar. Una segunda línea de políticas privatizadoras, complementaria a las ya mencionadas políticas que inciden sobre la oferta, fue orientada a financiar directamente la demanda (vía ayudas directas o incentivos fiscales), dotándola de una mayor capacidad de elección ante una oferta cada vez más diversa.

Todo esto va configurando un nuevo entramado institucional que ha venido en llamarse como welfare mix (Evers, 1991; Evers y Svetlik, 1993), mixed economy of welfare o welfare pluralism (Jonhson, 1999). Estos sistemas de bienestar, que aunque en opinión de Johnson (1999) realmente siempre han sido mixtos, replantearán los elementos centrales de los sistemas de bienestar europeos: el estado deja de ser el proveedor principal de servicios y delega esas funciones sobre las entidades privadas tanto lucrativas como no lucrativas, pero al mismo tiempo mantiene una importancia central en todo lo referido a la financiación, la regulación y la inspección de estos servicios (Evers, 2005).

El cuestionar la centralidad del estado lleva a reconocer una pluralidad de actores, entre los cuales destacan dos que entre los teóricos del estado de bienestar apenas habían sido 
tenidos en cuenta: la esfera informal y el tercer sector (Johnson, 1999). Así, estas nuevas corrientes ponen en valor la labor desempeñada tanto en el ámbito doméstico como a través de entidades no lucrativas en la provisión de servicios de bienestar, haciendo un reconocimiento explícito de la aportación fundamental de estos dos sectores (Evers y Svetlik, 1993).

Por último, además de realizar un reconocimiento al carácter plural de los sistemas de bienestar, los teóricos del welfare mix establecen cuál debería ser el papel y el peso de cada uno de esos agentes en los sistemas de bienestar que van resultando de la crisis del estado de bienestar. Así, como tendencia generalizada, indican que el estado va a ir retirándose de la provisión directa de servicios en beneficio de las entidades privadas, pero se reforzará en su función de regulador, financiador y evaluador de la calidad del servicio prestado. El sector público pasa a ser el responsable en última instancia del servicio público prestado, por mucho que no haya participado directamente en la provisión. Y al mismo tiempo, en su función dinamizadora o posibilitadora, tratará de garantizar también la "libertad de elección" del usuario, abriendo por un lado la provisión de servicios públicos a la competencia de entidades privadas y dotando de solvencia a la persona usuaria a través de las políticas de demanda.

Más allá del valor discursivo de este carácter plural o mixto de actores, el hecho es que esta nueva reorganización se ha resuelto básicamente en un esquema de "rolling back the state" donde las entidades privadas, principalmente de carácter lucrativo, han ido ganando posiciones en detrimento de las entidades del tercer sector.

\subsection{Alternativas a la privatización: coproducción, gobernanza y coconstrucción}

La crisis del estado de bienestar y la progresiva configuración de modelos mixtos (o privatizados) en los servicios públicos ha derivado en una profusa literatura sobre distintas formas de entender esta colaboración entre agentes públicos y privados.

Una de las primeras aproximaciones fue la que adoptaron los teóricos americanos en gestión pública en los años 70 y 80 bajo el concepto de co-producción (Parks, et al, 1999). En aquella época el debate en torno a la forma más eficiente de proveer servicios públicos estaba muy dominado por los que sostenían la necesidad de crear estructuras centralizadas y burocráticas. Sin embargo, a través de la propuesta de co-producción, esto autores defendían que el sistema más eficiente era aquel que posibilitara que la sociedad civil pudiera producir, al menos en parte, los servicios que iba a consumir. 
Esta primera aproximación tenía una visión micro, centrada en el rol de los individuos o grupos de individuos en la producción de dichos servicios (Brandsen y Pestoff, 2008). Sin embargo en el Reino Unido, la co-producción ha sido usada más recientemente para estudiar el papel de las entidades voluntarias o comunitarias en la provisión de servicios públicos (Osborne y McLaughlin, 2004). Y desde un enfoque más europeo, la co-producción también se ha solido referir a la creciente implicación organizada de la ciudadanía en la producción de sus propios servicios de bienestar (Pestoff, 2005). Todos ellos concluyen que la participación del tercer sector (entendido éste como grupos de ciudadanos o grupos de organizaciones) transforma la forma de proveer los servicios públicos, al mismo tiempo que el tercer sector también es transformado por el propio servicio que provee (Brandsen y Pestoff, 2008).

Pero más allá de concebir esta acción colectiva de la ciudadanía en la prestación de los servicios, hay quien analiza la participación de la sociedad civil desde episodios anteriores a la provisión, es decir, en las fases de planificación, diseño o articulación de los servicios. Siguiendo la clasificación propuesta por Osborne y McLaughlin (2004) cabría distinguir al menos tres formas de cooperación entre tercer sector y sector público:

- la co-gobernanza, donde el tercer sector participa en la planificación y la provisión del servicio

- la co-gestión, donde el tercer sector colabora junto con el estado en la provisión de servicios

- la co-producción, en sentido estricto, significa que los ciudadanos producen al menos en parte sus propios servicios.

Esta clasificación permite identificar dos variables de análisis: el carácter colectivo o individual de la relación, por un lado, y la fase del ciclo político, por otro. Así, con respecto a la primera, la co-producción se referiría a una participación de carácter individual mientras que las otras son interacción entre organizaciones (públicas y privadas). Y en lo que se refiere a la fase, se distingue entre las fases de planificación y de producción.

En esta misma línea se encuentra la aportación del concepto de co-construcción en oposición al concepto de co-producción. Tal y como establece Vaillancourt (2011: 40): “...En suma, la coproducción de las políticas públicas se desarrolla sobre el plano organizacional (en la organización de productos y servicios), mientras que la co-construcción se desarrolla sobre el plano institucional (en la fijación de orientaciones generales y de elementos 
fundadores de la política”. Esta primera noción de co-producción es muy próxima también a la noción de welfare mix o mixed economy of welfare analizado anteriormente (Evers, 1991,1993; Pestoff, 1999; Johnson, 1999). Por lo tanto, la noción de co-construcción iría más allá, tal y como dice Vaillancourt (2011: 43): "la co-construcción se relaciona con las políticas públicas en el momento de su elaboración y no solamente en el momento de su implementación”.

Vaillancourt (ibid.) distingue cuatro tipos o modelos de co-construcción: i) una primera, denominada mono-construcción, será aquel en el que el estado no comparte, sino que es el único protagonista en la toma de decisiones; ii) co-construcción neoliberal, (de moda en muchos países, notablemente con la popularidad de la corriente dominante de la New Public Management), donde el Estado es motivado para construir políticas públicas en cooperación con los actores socioeconómicos dominantes de la economía de mercado; iii) co-construcción corporativista, ciertos sectores de actividad socioeconómica y actores ligados al mundo patronal y sindical están incluidos en el diálogo y en la deliberación con el Estado, mientras que otros son excluidos, y por último, iv) la co-construcción democrática y solidaria.

Este último modelo de co-construcción se caracteriza de la siguiente forma: i) el Estado permanece como un socio diferente a los otros, dialoga, interactúa y delibera con los actores no estatales, permanece a la vez "sobre" y "próximo" a ellos; ii) el Estado llegar a ser partenaire de la sociedad civil, sin por ello dejar de serlo de los actores de la economía de mercado (se inscribe dentro de una perspectiva de economía plural); iii) implica una deliberación entre lo mejor de la democracia representativa y de la democracia participativa, e; iv) implica el reconocimiento de la participación de los actores de la economía social, al igual que una relación de tipo partenarial entre el Estado y esos actores (Vaillancourt, 2011).

Por considerar la economía social como principal agente aliado, este modelo de "coconstrucción democrática y solidaria" coincide plenamente con el modelo de "gobernanza de tipo partenarial" propuesto por Enjolras (2008). Este autor contrapone este modelo al actualmente hegemónico, que denomina como "gobernanza concurrencial", centrado en la extensión de los mecanismos de regulación de mercado (Enjolras, 2008:19).

Ante este modelo, expuesto anteriormente bajo la denominación del New Public Management, Enjolras antepone el modelo partenarial, donde el sector público no desempeña tanto un papel coercitivo, sino más bien de coordinador entre distintos agentes de la sociedad 
civil y el propio estado. El ámbito local aparece aquí como espacio privilegiado, al ser el espacio donde el capital social posibilita desarrollar los lazos institucionales y la puesta en valor del territorio. En esta red de agentes la economía social (y solidaria) juega un papel central en la puesta en marcha de este modelo de gobernanza, al valorizar lo local en lo territorial, y lo democrático en lo organizacional.

Una vez de realizar la revisión de los modelos teóricos de relación entre el estado y la economía social, pasamos a presentar los resultados obtenidos en el estudio de caso que se ha realizado en el territorio histórico de Gipuzkoa. Este estudio ha tratado de cotejar la opinión de una serie de expertos en temas de política social sobre la estrategia de cooperativizar el sector de los servicios sociales.

\section{ESTUDIO DE CASO: LA COOPERATIVIZACIÓN DE LOS SERVICIOS SOCIALES EN EL TERRITORIO HISTÓRICO DE GIPUZKOA}

Tal y como se ha ido adelantando hasta el momento, el estudio de campo que complementa esta contribución ha tenido como objetivo plantear como hipótesis de trabajo a los propios responsables del Departamento de Política Social de la Diputación Foral de Gipuzkoa el ir cooperativizando progresivamente la gestión de los servicios sociales. La cooperativización de los servicios sociales se plantea como hipótesis de trabajo, en el sentido de que esta estrategia aún no ha sido ampliamente desplegada por los responsables del departamento, aunque a un nivel ideológico puedan mostrar cierta simpatía con respecto a ella.

Por lo tanto, se ha tratado de enfocar el estudio en el sentido de indagar si, desde su perspectiva y posición, serían partidarios de que el departamento promoviese de forma activa (bien facilitando su creación o bien formando parte de las mismas) la creación de nuevas cooperativas, o la reconversión de las empresas actualmente existentes en cooperativas. Más que en aspectos técnicos que posibilitarían esta estrategia, el cuestionario ha tratado de incidir sobre el enfoque ideológico de estos responsables, cotejando si estos escenarios serían deseables en su opinión, más allá de que puedan mostrar ciertas dificultades tanto técnicas, legales como políticas.

Se trataría, en definitiva, de tratar de teorizar sobre si los encargados de diseñar la política social en Gipuzkoa son partidarios de canalizar la participación ciudadana a través de 
entidades del tercer sector/economía social ${ }^{2}$, y en tal caso hasta que nivel de profundización debería darse esta implicación.

\subsection{Apuntes metodológicos}

El objetivo del estudio Delphi que se presenta a continuación es presentar la opinión de los distintos responsables del Departamento de Política Social de la Diputación Foral de Gipuzkoa con respecto a la estrategia de cooperativizar la gestión de los servicios sociales de su territorio.

Se ha optado por aplicar la metodología Delphi, al ser ésta una técnica de investigación social que posibilita, partiendo de un grupo de expertos, obtener una opinión grupal lo más fidedigna posible. Se trata de un método iterativo (cada experto es preguntado por la misma pregunta al menos dos veces), que respeta el anonimato de los participantes y que cuenta con un feedback controlado, ya que es el coordinador del estudio quien realiza la devolución de las aportaciones más importantes, evitando la información irrelevante (Landeta, 1999). El objetivo último de esta técnica es intentar acercar las posiciones iniciales de los expertos, de cara a llegar a una opinión grupal lo más consensuada posible, pero realizando la encuesta de forma personalizada para intentar evitar dinámicas de grupo que anulan o ejercen cierta presión sobre la opinión de cada participante.

Para ello, ha sido necesaria la elaboración de un panel de expertos. Se ha acotado el estudio al territorio histórico Gipuzkoano debido a que las instituciones públicas con mayor grado de responsabilidad en la gestión de los servicios sociales a nivel de Euskadi son las propias diputaciones forales. Optando por la más cercana, la Gipuzkoana, se ha contactado con expertos que además de contar con un conocimiento detallado del sector, tienen capacidad de influencia sobre el mismo, al ser la mayor parte de ellas decisores públicos. Este estudio Delphi ha contado con la participación inicial de 16 personas, 11 de ellos respondiendo a un perfil político (cargos políticos electos, la mayoría desempeñando una función de dirección o asesoramiento de los principales ejes de actuación del Departamento de Política Social) y los 5 restante con un perfil más técnico (todos ellos jefes de servicios de

\footnotetext{
${ }^{2}$ En este apartado utilizaremos indistintamente los términos "economía social" y "tercer sector". En el cuestionario se ha empleado el término tercer sector, al considerar que en los servicios sociales la economía social como término no goza de mucha popularidad, y únicamente se suele asociar a cooperativas. Por el contrario, el tercer sector también integra ciertas cooperativas, aquellas exentas de fines lucrativos. Por ello, cuando hablemos de tercer sector lo haremos en sentido amplio, aquel que integra también las cooperativas, y cuando hablemos de economía social también nos referiremos al conjunto de entidades no lucrativas.
} 
su área). De los 16 expertos que iniciaron el proceso, 13 finalizaron todo el proceso de forma satisfactoria.

La realización de la encuesta se ha basado en una batería de 26 preguntas, clasificados en 5 bloques, casi todas a responder con una escala Likert del 1 al 5 (donde 1 es total desacuerdo, 5 total acuerdo y 3 ni a favor ni en contra). Se ha realizado un esfuerzo importante en formular las preguntas de forma clara y comprensible, adaptando el lenguaje utilizado desde un registro académico a otro de utilización más común. Por último, cada bloque de preguntas se cierra con una pregunta abierta para que los expertos dispongan de un espacio para escribir cualquier aportación relacionada con las preguntas previas.

De esta forma se trata de combinar los resultados cuantitativos con los cualitativos. Por un lado se han objetivado las respuestas a través de medias y desviaciones típicas, y se ha analizado el nivel de aproximación entre la primera ronda y la segunda mediante la comparación de estas dos variables. Por otro lado, en caso de que las respuestas no muestren un consenso claro ni se perciban movimientos significativos de aproximación entre expertos, el análisis de las aportaciones ha posibilitado la búsqueda de algunos argumentos que justifiquen la naturaleza de las respuestas obtenidas.

El proceso de investigación cuyos resultados mostramos a continuación ha constado de tres fases. En una primera fase se organizó un Focus Group donde participaron distintos responsables del departamento junto con agentes sociales e investigadores, y se detectaron las variables más significativas y las que más debate planteaban en torno al objeto de estudio propuesto. Una vez de elaborar el cuestionario que recogía dichas variables y de contactar con los participantes del panel de expertos, en la segunda fase los expertos fueron solicitados a responder el cuestionario dos veces en dos rondas consecutivas, sabiendo en la segunda ronda cuáles habían sido las medias obtenidas en la primera para cada pregunta y la aportaciones más significativas realizadas por los expertos (feedback controlado). El proceso de investigación se dio por terminado con un seminario organizado por los propios autores del estudio, donde se hizo delante (de parte) de los participantes la devolución completa de los resultados que desgranamos a continuación. 


\subsection{Principales resultados}

\subsubsection{Modelo de gestión}

Un primer bloque de preguntas analizaba el posicionamiento de los expertos en cuanto al modelo ideal de servicios sociales, donde podían optar por un modelo estrictamente público, estrictamente privado o un modelo concertado.

Cabría señalar tres evidencias entre los resultados obtenidos: i) la absoluta mayoría se muestra contraria al modelo privado (92\%); ii) una mayoría contundente (62\%) se muestra partidaria del modelo público, y; iii) una cantidad importante de expertos no descarta el modelo concertado (38\%), al considerar que es éste el que realmente predomina a día de hoy en Gipuzkoa. Sin embargo, las respuestas en cuanto a la idoneidad del modelo concertado muestran una dispersión importante, ya que se distribuyen más o menos en la misma proporción los expertos partidarios y los detractores (31\%), habiendo un tercer tercio de expertos que no toman partido.

El proceso de aproximación entre expertos no ha alterado de forma significativa las respuestas iniciales, únicamente es reseñable una mayor concentración de opiniones contrarias al modelo privado en la segunda ronda.

En cuanto al análisis cualitativo, gran parte de las aportaciones subrayan la importancia de mantener estos servicios bajo responsabilidad pública, aunque luego el modelo de gestión no sea compartido al mismo nivel. Ha habido expertos que han puesto en valor la aportación de las entidades del tercer sector en la gestión de estos servicios, y ha habido quien considera que el modelo de gestión no es determinante en la calidad final de los servicios.

\subsubsection{Modelo de co-construcción}

Un segundo bloque de preguntas incidía en la cuestión del nivel de participación de la economía social en la toma de decisiones en cuanto al diseño mismo de los servicios sociales. Las preguntas abarcaban tres tipos de modelos de participación: el informativo, el consultivo y el decisorio. En el modelo informativo el tercer sector únicamente es tenido en cuenta a la hora de dar cuenta de las decisiones adoptadas. En el nivel consultivo su opinión es integrada en la decisión final, al considerar que el tercer sector es un agente con voz autorizada en el sector. En el tercer nivel, el tercer sector es co-decisor junto con la administración pública, lo que podría ser equiparado al modelo de co-construcción teorizado anteriormente. 
Los resultados obtenidos en este apartado son los siguientes: i) una mayoría importante $(76 \%)$ se muestra contraria a que el tercer sector sea tenida en cuenta únicamente a un nivel informativo; ii) en cuanto al modelo consultivo, este modelo no llega a convencer del todo a casi nadie, ya que casi la mayoría de expertos se sitúa en los valores intermedios, es decir no hay una mayoría amplia ni que la respalde, ni que la descarte; iii) sin embargo, más de la mitad de expertos (54\%) ve con buenos ojos la co-construcción, siempre y cuando la última palabra sea la de la administración pública. Es de destacar que existe también un porcentaje importante de expertos contrarios a este último aspecto.

El análisis del grado de aproximación de los expertos arroja resultados interesantes en este bloque, ya que se percibe claramente que la opinión de los participantes ha ido posicionándose hacia el modelo consultivo y alejándose del modelo de co-construcción.

Este proceso puede haberse visto motivado por las aportaciones realizadas, ya que muchas de ellas hacen hincapié en que la decisión en caso de disparidad de opiniones o en caso de confrontación de intereses debe, en última instancia, recaer sobre los cargos políticos, ya que son éstos los responsables últimos de la calidad de los servicios prestados. En este sentido, cabe señalar que la participación del tercer sector y otros agentes sociales también despierta ciertos recelos en algunos expertos, al considerar que este espacio también puede dar cabida a una serie de intereses privados poco compatibles con el interés común.

\subsubsection{Formalización de la gestión indirecta}

Un tercer bloque de preguntas hace referencia a la manera en que se debería formalizar la relación entre la administración pública como contratante (y responsable) del servicio y la economía social como gestor o proveedor del mismo. Se han barajado tres fórmulas distintas: la concertación, en el cual la administración respalda económicamente un servicio de titularidad privada; la contratación, donde la administración saca a concurso la gestión de un recurso de titularidad pública, y; el convenio, a través del cual la administración establece de forma discrecional un compromiso financiero con una entidad privada para la prestación de un servicio en concreto.

El resultado general de este bloque es que los expertos no tienen claro cuál de estas tres fórmulas debería ser la norma general. Ninguna de estas tres opciones recaba mayorías suficientes ni a favor ni en contra. Más de la mitad de las opiniones se sitúa en el punto 
intermedio en las tres preguntas $(54 \%, 62 \%, 54 \%$, respectivamente), y apenas se sitúa opinión alguna en los valores extremos (totalmente de acuerdo o totalmente en desacuerdo).

El nivel de aproximación entre expertos no hace más que reforzar esta indeterminación: en el modelo de concertación, así como en el de contratación y en el de convenio, las alteraciones que se aprecian entre rondas son todas tendentes hacia el punto intermedio, alejándose desde posicionamientos totalmente favorables y desfavorables. Ha podido haber una especie de consenso, por tanto, a la hora de suavizar las respuestas iniciales.

Esta falta de posicionamiento claro en la escala Likert hace que el análisis cualitativo sea fundamental para la comprensión de estos resultados. Gran parte de los expertos señala que la naturaleza del servicio a prestar es el que determina la manera de formalizar esta relación, bien a través de concierto, contrato o convenio. No existe un modelo perfecto válido para todo tipo de servicio. Sin embargo, hay quien opina que la contratación es la fórmula que más garantías ofrece, aunque la ley de servicios sociales apueste por un modelo de concertación de cara futuro. El convenio debería de reservarse según otro participante únicamente para aquellos servicios que no estén contemplados en el catálogo de servicios (y, por lo tanto, que no son de responsabilidad pública). Por último, hay quien resta importancia a este aspecto, y señala que lo importante es tener bien definidos los objetivos y el catálogo de servicios.

\subsubsection{Medidas de discriminación positiva}

Un cuarto bloque pone sobre la mesa la utilización de medidas de carácter discriminatorio a favor de las entidades del tercer sector en su conjunto, y de las formas cooperativas en concreto.

Esta pregunta ha obtenido respuestas meridianamente claras: i) una amplia mayoría (69\%) está a favor de discriminar de forma positiva al tercer sector frente al sector privado capitalista; ii) una mayoría aún mayor (85\%) cree que las cláusulas sociales son la herramienta más eficiente para aplicar este tipo de medidas, y; iii) una mayoría consistente (61\%) también opina que la fórmula cooperativa es la que más especialmente se debería de preservar, aunque este extremo también aglutina a un porcentaje importante en contra (31\%).

El análisis de la aproximación de los expertos también refuerza esta sensación de consenso en las tres respuestas, ya que no se han alterado mucho los posicionamientos 
favorables en las dos primeras cuestiones, y en la idoneidad de las cooperativas esta posición partidaria se fortalece en la segunda vuelta.

Sin embargo, consideramos que las aportaciones de los expertos en este caso son especialmente interesantes, ya que matizan en cierta forma el supuesto consenso que reflejan los resultados cuantitativos. Así, la mayor parte de los comentarios señalan que lo importante, más allá de la figura jurídica, es la calidad de los servicios prestados, y una vez garantizado esto, entonces cobraría sentido el empleo de medidas discriminatorias. Otro número de comentarios apuntan en el sentido de advertir que la figura jurídica no es garantía de nada, ni de que el servicio cuente con la calidad necesaria, ni de que estas empresas funcionen realmente de forma democrática y no lucrativa.

Por lo tanto, como síntesis del análisis cuanti/cualitativo, se concluye que la mayoría se posiciona a favor de medidas discriminatorias, pero no en beneficio de unas figuras jurídicas concretas, o más bien a favor de este tipo de empresas una vez que éstas hayan certificado las capacidades técnicas y organizativas necesarias para la prestación del servicio en cuestión.

\subsubsection{Estrategia de cooperativización}

El último bloque se reserva para la validación por parte de los expertos de la estrategia de cooperativización de los servicios sociales, planteado como hipótesis de trabajo, ya que esta estrategia no ha tenido una implantación considerable en el territorio hasta el momento.

Esta validación pivota sobre tres cuestiones fundamentales: i) considerar si este proceso de cooperativización va a favor o en detrimento del carácter público de los servicios sociales; ii) valorar si la cooperativización es un objetivo en sí mismo, o un paso intermedio hacia otro escenario distinto (o bien hacia un modelo publificado u otro privatizado), y; iii) identificar cual sería el modelo más adecuado de cooperativizar el sector.

El resultado global de este bloque es que los expertos muestran dudas y desavenencias importantes con respecto a estas tres cuestiones.

En cuanto a la estrategia de cooperativización como vía para aumentar el control público y social, las opiniones son muy dispersas, repartidas de forma casi igualitaria entre partidarios, detractores y neutrales. Una distribución parecida es la que se obtiene de la pregunta de si la cooperativización es una manera de privatizar los servicios sociales, ya que

REVESCO No 120 - Primer Cuatrimestre 2016 - ISSN: 1885-8031 - www.ucm.es/info/revesco 
aunque un mayor porcentaje de expertos niega esta hipótesis (46\%), también hay una parte importante que lo subscribe $(31 \%)$ o no lo descarta.

En cuanto a si es un objetivo deseable o no, tampoco se obtienen resultados muy claros. La mayoría de expertos considera que puede ser una opción (69\%), aunque no para aliviar problemas puntuales de gestión (16\%), ni como objetivo intermedio hacia la publificación del sistema en su conjunto (16\%). Pero tampoco es considerado como un fin en sí mismo, como un modelo deseable hacia el que deberían orientarse las políticas sociales, ya que sólo un tercio se posiciona de forma positiva sobre este aspecto.

Por último, en cuanto al modelo preferido para cooperativizar el sector, se ha dado a elegir entre cuatro modelos distintos: crear cooperativas de trabajo asociado (sin ninguna característica adicional), promover cooperativas de utilidad pública, impulsar cooperativas de iniciativa social, o formar parte en el capital social de cooperativas mixtas ${ }^{3}$. El modelo que más respaldo obtiene es el de las cooperativas de iniciativa social (3,91 de media en la segunda ronda), seguido de las cooperativas mixtas $(3,33)$, las de utilidad pública $(3,18)$ y por último las cooperativas corrientes $(2,16)$.

Este orden de preferencias podría señalar ciertas características que los expertos consideran como garantías fundamentales del proceso de cooperativización de los servicios sociales: i) que estas cooperativas sean no lucrativas; ii) que sean compatibles con un mayor control público, y; iii) que sean consideradas, al igual que las cooperativas de enseñanza en el sector educativo, como partes integrantes del modelo público-concertado de servicios sociales.

\section{CONCLUSIONES FINALES}

Como conclusiones finales del trabajo presentado señalaríamos las siguientes.

Se ha visto a través de la revisión de la literatura que la mayor implicación de la economía social en el diseño y la provisión de los servicios sociales podría ser considerada como una estrategia válida para intentar socializar (que no privatizar) los servicios públicos,

\footnotetext{
${ }^{3}$ Las tres primeras opciones son cooperativas puramente privadas, donde el capital social es propiedad exclusiva de los socios-trabajadores. La segunda y la tercera son reconocimientos legales que otorga el Gobierno Vasco a cooperativas que desempeñan una función pública y a las cooperativas sin ánimo de lucro, respectivamente. Y por último, la fórmula de la cooperativa mixta, aunque desconocida hasta ahora a nivel de Euskadi, sería aquella que permitiría la participación financiera del sector público (como socio colaborador o en la forma que se determine) en la propia cooperativa.
} 
es decir, para intentar abrirlos a la participación ciudadana y a la autogestión. Así, se han apuntado propuestas teóricas que, más allá del carácter individual de la participación ciudadana (Co-producción), valorizan la dimensión colectiva de esta acción a través de entidades de la economía social. La "co-construcción democrática y solidaria” propuesta por Yves Vaillancourt (2011), o la "gobernanza de tipo partenarial" teorizada por Bernard Enjolras (2008), podrían ser dos referentes teóricos a tener en cuenta en esta dirección.

Trasladando esta inquietud a un ámbito territorial concreto, a saber, el Departamento de Política Social de la Diputación Foral de Gipuzkoa, se ha desarrollado un estudio Delphi sobre la posibilidad de ir cooperativizando progresivamente el sector de servicios sociales, cuyos resultados principales serían los siguientes:

- El departamento es más proclive a un modelo público que a otro concertado, con una premisa ideológica bien clara: que la responsabilidad de la calidad de los servicios prestados en última instancia es pública y en ningún caso privatizable.

- En cuanto al modelo de co-construcción, el departamento considera muy importante la participación de la economía social en el diseño de los servicios sociales pero a un nivel básicamente consultivo, donde los responsables públicos se reservan la potestad exclusiva de decisión en caso de desavenencias o conflicto de intereses.

- En cuanto a la manera de formalizar la gestión indirecta el departamento opina que cada tipo de servicio requiere de un marco distinto de relaciones (sea concierto, contrato o convenio), por lo que no se apuesta decididamente por generalizar ninguna de ellas para el conjunto del sistema.

- En lo que se refiere a las medidas de discriminación positiva, destacar que el departamento valora muy positivamente la incorporación de cláusulas sociales en la contratación pública, si bien estas cláusulas deberían aplicarse entre las entidades que en primer lugar certifiquen suficiencia técnica, para intentar favorecer y preservar ciertas prácticas, más que figuras jurídicas concretas (como puedan ser las no lucrativas o cooperativas).

- Por último, en cuanto a la hipótesis de trabajo de ir cooperativizando el sector de los servicios sociales destacar que, aunque la propuesta haya tenido buena acogida, a día de hoy genera algunas dudas e incertidumbres en el seno del departamento. Aunque la mayoría no equipara este proceso con un proceso privatizador, tampoco se interpreta como una forma válida de expansión del modelo público. Además, se 
estima que la estrategia de cooperativización debería ir más allá de solucionar situaciones concretas, aunque tampoco se afirma que sea un objetivo final en sí mismo. Y para acabar, entre los modelos preferidos de cooperativización, se apuesta por aquellas que impulsen el carácter no lucrativo de las cooperativas y que garantice un mayor control por parte de los decisores públicos.

En cuanto a las líneas futuras de trabajo, parece interesante seguir investigando en las decisiones estratégicas que vaya tomando el departamento, ya que muchas de ellas van perfilando un horizonte distinto del esbozado hasta el momento. En un territorio donde la concertación con el tercer sector ha solido ser considerado como el modelo idóneo, el actual equipo de gobierno apunta hacia una re-publificación de los servicios, tal y como atestigua la reciente creación de un organismo autónomo foral (Kabia) que irá asumiendo la gestión de las residencias municipales. En opinión de los responsables políticos, este organismos pretende ser "un importante instrumento para avanzar hacia un cambio de modelo orientado a la gestión directa de los servicios sociales en Gipuzkoa"4 . Es por ello que la cooperativización de la gestión de los centros de servicios sociales no parece por el momento ser la apuesta política más decidida del Departamento de Política Social, aunque tal y como se ha visto en el estudio, los posicionamientos favorables en cuanto a la incorporación de cláusulas sociales en la contratación pública podrían abrir espacios para que ambos objetivos puedan ser complementarios y compatibles.

\section{BIBLIOGRAFÍA}

ASCOLI, U. y RANZI, C.(ed.), (2002) Dilemmas of the Welfare Mix: the newstructure of welfare in an era of Privatization, Kluwer Academic/Plenum Publishers, New York.

BRANDSEN, T. y PESTOFF, V. (2008) An Introduction, in V. PESTOFF y T. BRANDSEN (Eds), Co-production.The third sector and the delivery of public services (pp. 1-9). Oxon: Routledge.

ENJOLRAS, B. (2008) Gouvernance et intérêt général dans les services sociaux et de santé, Bruxelles, Bern, Berlin, Frankfurt am Main, New York, Oxford, Wien, Peter Lang (Collection: Économie sociale \& Économie publique/Social Economy \& Public Economy).

EVERS, A. (1991) Shifts in the welfare mix, Eurosocial, $n^{\circ} 57 / 58$, pp. 7-8.

\footnotetext{
${ }^{4}$ http://www.noticiasdegipuzkoa.com/2014/07/14/sociedad/euskadi/diputacion-de-gipuzkoa-asumira-la-gestionde-las-residencias-municipales-a-traves-del-organismo-autonomo-kabia (consultado el 17-07-2014)
} 
EVERS, A y SVETLIK, I. (1993) Balancing pluralism: new welfare mixes in care for the elderly, London, Avebury.

EVERS, A. (2005) Mixed Welfare Systems and Hybrid Organizations: Changes in the Governance and Provision of Social Services, International Journal of Public Administration, vol. 28, $\mathrm{n}^{\circ}$ 9, pp. 737-748, Taylor \& Francis Inc.

JOHNSON, N. (1999) Mixed Economies of Welfare: a Comparative Perspective, Pearson Education, Prentice Hall Europe, Hertfordshire.

LANDETA, J. (1999) El método Delphi. Una técnica de previsión del futuro. Barcelona: Ariel Social.

OSBORNE, S. y MCLAUGHLIN, K. (2004) The Cross-Cutting Review of the Voluntary Sector: Where Next for Local Government Voluntary Sector Relationships?, Regional Studies, vol. 38, n 5 , pp. 573-82.

OSTROM, E. (1999) Crossing the Great Divide: Coproduction, Synergy, and Development, in M. MCGINNIS (ed.) Polycentric Governance and Development: Readings from the Workshop in Political Theory and Policy Analysis. Ann Arbor, MI: University of Michigan Press.

PARKS, R.B., BAKER, P.C., KISER, L., OAKERSON, R., OSTROM, E., OSTROM, V., PERCY, S. L., VANDIVORT, M. B., WHITAKER, G. P. y WISON, R. (1991) Consumers as Co-Producers of Public Services: Some Economic and Institutional Considerations, Policy Studies Journal, vol. 9, n 7, pp. 1001-11.

PESTOFF, V. (1998, 1999, 2000) Beyond the Market and State. Social Enterprises and Civil Democracy in a Welfare Society; Ashgate eta English Editions. Singapore, Sydney eta Tokyo.

PESTOFF, V. (2005) The Third Sector and the Democratization of the Welfare State: Revisiting the Third Sector and State in Democracy and Welfare Theory; Östersund: Research Report.

PESTOFF, V., OSBORNE, S. P. y BRANDSEN, T. (2008) Patterns of Co-production in Public Services, in V. PESTOFF y T. BRANDSEN (Eds), Co-production.The third sector and the delivery of public services (pp. 99-103). Oxon: Routledge.

VAILLANCOURT, Y. (2011) La economía social en la coproducción y la coconstrucción de las políticas públicas, Revista del Centro de Estudios de Sociología del Trabajo, n³, pp. $31-68$.

WALZER, M. (1988) Socializing the Welfare State, in A. GUTMANN (ed.) Democracy and the Welfare State. Princeton, NJ: Princeton University Press. 